\title{
Effect of Bottom Baffles and Air Inlet Position on Mass Transfer Performance of a Water-Sparged Aerocyclone
}

\author{
Facheng Qiu ${ }^{1}$, Wen Cheng ${ }^{1}$, Haifeng $W u^{1}$, Fei Xu ${ }^{1}$, Xuejun Quan ${ }^{1,}$, , Lu Yang ${ }^{2}$ \\ ${ }^{1}$ School of Chemistry and Chemical Engineering, Chongqing University of Technology, Chongqing, China \\ ${ }^{2}$ Chongqing Municipal Solid Waste Resource Utilization \& Treatment Collaborative Innovation Center, Chongqing, China
}

Email address:

hengjunq@cqut.edu.cn (Xuejun Quan)

${ }^{*}$ Corresponding author

\section{To cite this article:}

Facheng Qiu, Wen Cheng, Haifeng Wu, Fei Xu, Xuejun Quan, Lu Yang. Effect of Bottom Baffles and Air Inlet Position on Mass Transfer Performance of a Water-Sparged Aerocyclone. American Journal of Chemical Engineering. Vol. 6, No. 4, 2018, pp. 44-48. doi: 10.11648/j.ajche.20180604.11

Received: June 20, 2018; Accepted: July 1, 2018; Published: July 25, 2018

\begin{abstract}
Water-sparged Aerocyclone (WSA) is a new type of gas-liquid mass transfer equipment with a coupling field of liquid jet with gas cyclone, which can be widely used in wastewater treatment process. To further optimize the structural design of WSA, the effect of bottom baffles and air inlet position on mass transfer performance of WSA was comparatively studied by air stripping of ammonia from wastewater. The results indicated that the separation space configuration of a WSA affects its mass transfer performances. Under the same condition, the axial air inlet position has no effect on mass transfer performance, but moving air inlet position downward could reduce the gas pressure drop in WSA by about $10 \%$, which was probably caused by abating the friction loss between the gas cyclone and the wall. In case of high air inlet velocity and low liquid flow rate, the bottom baffles in the WSA could intensify the mixing effect between gas and liquid phases, thereby improving the mass transfer performance, and the effect is more pronounced with the increase of air inlet velocity. The results could be used as a guide for the design of WSA with excellent mass transfer performance.
\end{abstract}

Keywords: Cyclone, Baffle, Mass Transfer, Wastewater, Gas-Liquid Flow

\section{Introduction}

The crushing atomization of the liquid jet in the air stream is commonly used in engineering to reduce the atomization particle size of the liquid droplets and is widely applied in various mechanical systems such as aero engines, liquid rocket engines, and diesel engines [1-3]. The use of jet atomization to reduce the droplet size and increase the gas-liquid two-phase contact area is also a way to strengthen the mass transfer process in the chemical industry. The WSA is the use of liquid jet atomization in a three-dimensional gas-phase swirling field to form a liquid jet and gas swirling coupling field while using the statics of the swirling field [4]. The equipment has achieved good results in gas-liquid mass transfer and reaction, such as wastewater deamination, wet flue gas desulfurization and $\mathrm{Cr}$ (VI)-containing wastewater treatment [5-8].

The WSA currently under development is based on the structure of a traditional cylindrical cyclone separator. Its inlet port is located on the swirl head, and there is a certain axial distance from the liquid jet and gas swirl coupling area $[9,10]$. When the WSA is working, a certain height of the liquid seal is set at the bottom of the cylinder to ensure the main flow of internal air. In order to optimize the structure of these two zones, this paper examines the effects of the axial displacement of the inlet port and the setting of the baffle at the bottom of the main cylinder on the mass transfer performance of WSA.

\section{Material and Methods}

\subsection{Experimental Setup}

The experimental WSA is based on a traditional cylindrical cyclone. All the structures are made of transparent Plexiglas. 
The main basic design dimensions are shown in literature [11]. In this present work, in order to study the influence of inlet location and baffle on mass transfer effect, two different devices are adopted, as shown in Figure 1 and 2. In addition, four baffles with width of $D / 10$ vertically mounted on the bottom and inner wall. The thickness of the baffle is $3 \mathrm{~mm}$.

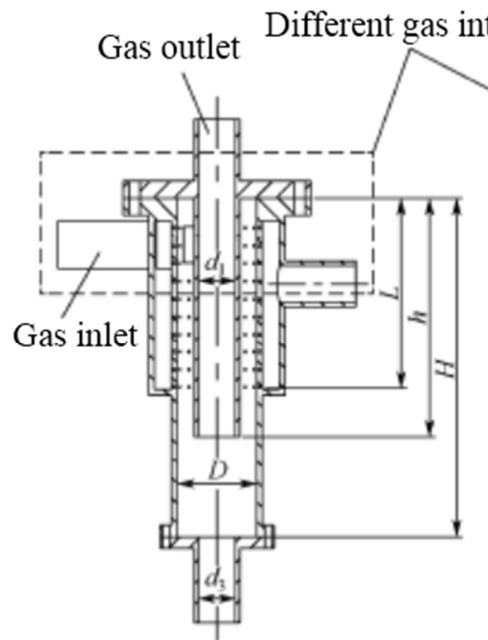

(a)

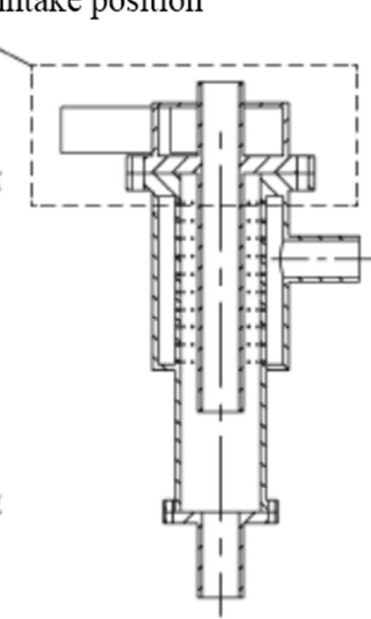

(b)
Figures. 1. Two different intake positions WSA schematic diagram.

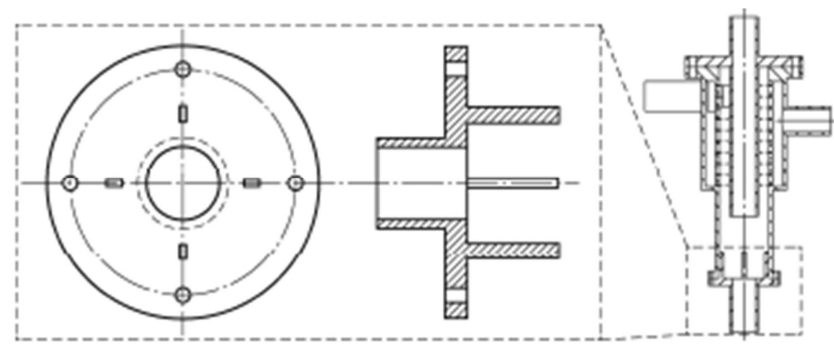

Figures 2. Schematic diagram of the setting and installation of the baffle.

\subsection{Experimental Method}

Air blowing ammonia water method was utilized to investigate the mass transfer performance in the experiment. The raw material was $5.8 \mathrm{~L}$ of ammonia solution with an initial ammonia nitrogen concentration of approximately $3500 \mathrm{mg} / \mathrm{L}$, and the amount of $\mathrm{NaOH}$ solution added during the entire stripping process was determined by calculating the theoretical consumption of $\mathrm{OH}^{-}$in the stripping process and blowing off experiments at the condition of $\mathrm{PH}>11$. The experiment adopts the intermittent operation mode, and adopts WSA with different structures to conduct experiments. The single experiment is performed for 60 minutes, the first 30 minutes are sampled every 5 minutes, and the rest 30 minutes are sampled every 10 minutes. Wastewater samples were measured for ammonia nitrogen concentration at $420 \mathrm{~nm}$ using Nessler's reagent spectrophotometry. The liquid circulation flow rate was set to $1.5 \mathrm{~m}^{3} / \mathrm{h}, 2.0 \mathrm{~m}^{3} / \mathrm{h}$, and 2.5 $\mathrm{m}^{3} / \mathrm{h}$, and blow-off experiments were carried out at different intake velocities.

The volumetric mass transfer coefficient of the deamination process is calculated as follows [12, 13]:

$$
-\ln \frac{c_{t}\left(\mathrm{NH}_{3}-\mathrm{N}\right)}{c_{0}\left(\mathrm{NH}_{3}-\mathrm{N}\right)}=K_{L} a \times t
$$

Where $c_{0}\left(\mathrm{NH}_{3}-\mathrm{N}\right)$ and $c_{t}\left(\mathrm{NH}_{3}-\mathrm{N}\right)$ are the ammonia nitrogen concentrations at the initial and $t$-times in water, respectively. $K_{L} a$ is volumetric mass transfer coefficient of ammonia. $t$ is the blowing time.

\section{Results and Discussion}

\subsection{Effect of Air Inlet Location}

As illustrated in Figures 3, the air inlet position has almost no effect on the mass transfer performance of the WSA at the same liquid-phase circulation flow rate and intake air velocity. This shows that the experimental design of the two air inlet positions has little effect on the jet-swirl coupling within the WSA. However, as can be seen from Figure 4, the inlet position has a significant effect on the gas-phase pressure drop in the WSA, and the WSA gas pressure drop of the air inlet axially moving down is lower than that of the traditional air inlet, and the value differs by more than $10 \%$. Indeed, the internal gas pressure drop of the WSA mainly is determined by the friction loss between the swirling field and the wall and the coupling between the internal gas swirling field and the liquid jet field. The air inlet axially moving down decreases the friction loss of gas-phase swirl flow and wall.

In order to further investigations of the influence of inlet location on WSA, the reflux ratio is taken into consideration. Results of the WSA internal reflux ratio of the two air intake structures are shown in Figure 5. The change of inlet position has little effect on reflux ratio, and the same rule of change was detected i.e. at higher liquid circulating flow rate, the reflux ratio increases with the gas inlet velocity. As the gas velocity increases, the liquid reflux ratio of the two structures decreases obviously with the increase in gas velocity. This is because of the formation of adherent atomization under low liquid-phase circulation flow. When the liquid phase enters the WSA through the jet orifice, most of the liquid phase is pressed against the inner wall of the main cylinder under the strong gas-phase swirling flow field. At the end, only a small part of the liquid phase is discharged from the central exhaust pipe by flowing out of the underflow port. Simultaneously, the liquid jet flow pattern exhibits an adherent atomized swirling jet, and the effective mass transfer area between two phases decreases. 


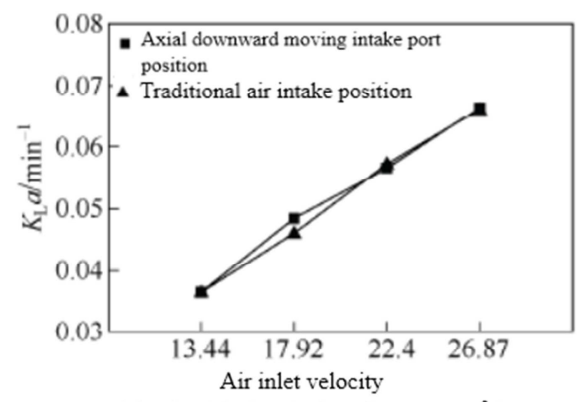

(a) Liquid circulation flow $1.5 \mathrm{~m}^{3} / \mathrm{h}$

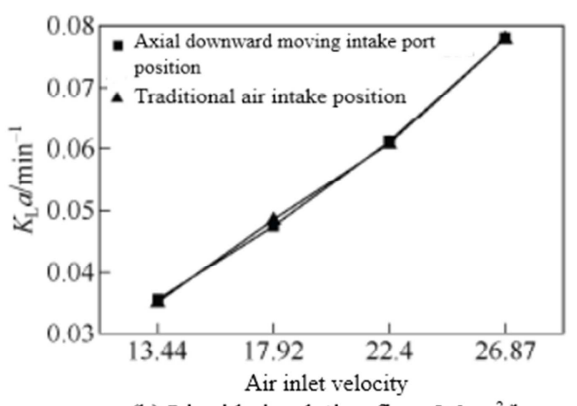

(b) Liquid circulation flow $2.0 \mathrm{~m}^{3} / \mathrm{h}$

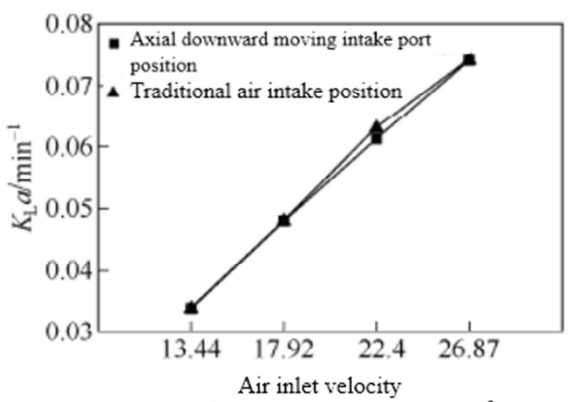

(c) Liquid circulation flow $2.5 \mathrm{~m}^{3} / \mathrm{h}$

Figure 3. Effect of inlet location on gas-liquid mass transfer coefficient in WSA under different liquid flow rates.

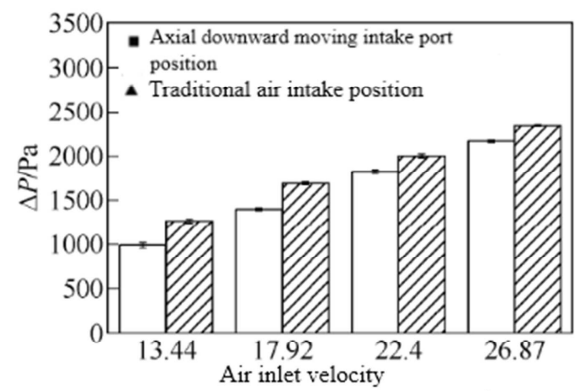

(a) Liquid circulation flow $1.5 \mathrm{~m}^{3} / \mathrm{h}$

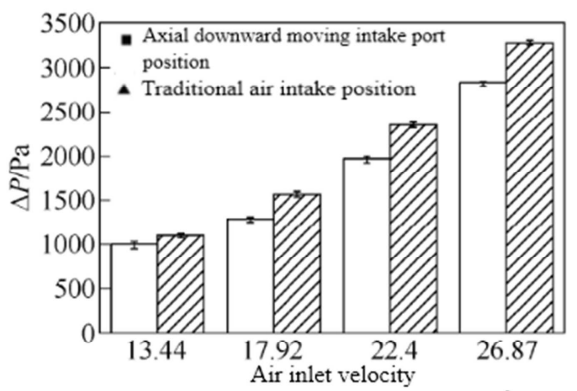

(b) Liquid circulation flow $2.0 \mathrm{~m}^{3} / \mathrm{h}$

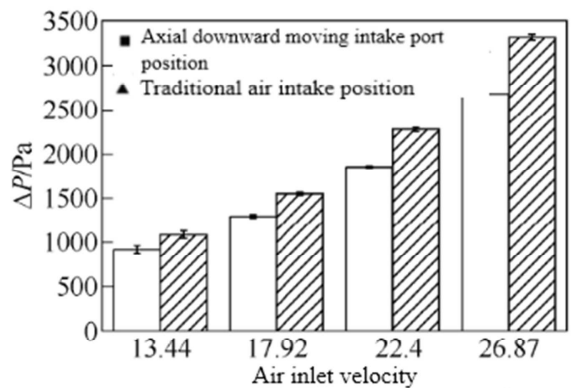

(c) Liquid circulation flow $2.5 \mathrm{~m}^{3} / \mathrm{h}$

Figures 4. Effect of inlet location on gas pressure drop in WSA under different liquid flow rates.

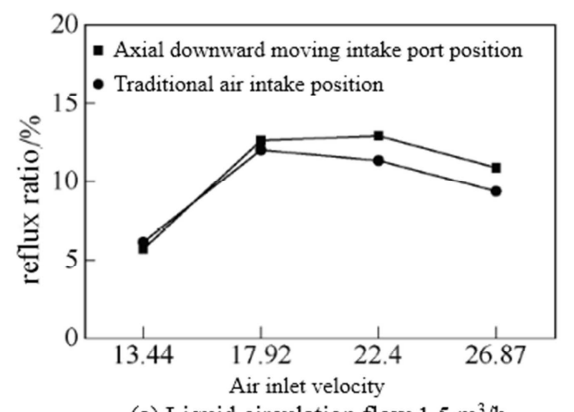

(a) Liquid circulation flow $1.5 \mathrm{~m}^{3} / \mathrm{h}$

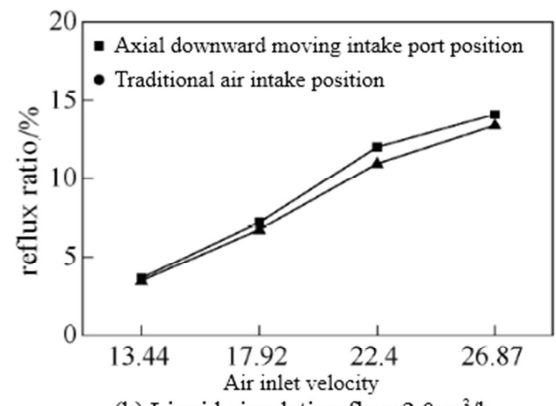

(b) Liquid circulation flow $2.0 \mathrm{~m}^{3} / \mathrm{h}$

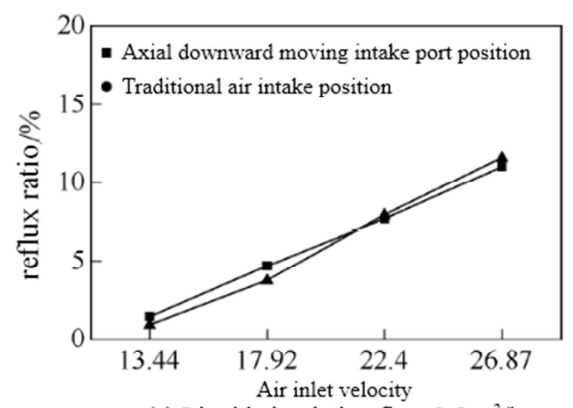

(c) Liquid circulation flow $2.5 \mathrm{~m}^{3} / \mathrm{h}$

Figure 5. Effect of inlet location on reflux ratio in WSA under different liquid flow rates.

\subsection{Effect of Bottom Baffle}

The effect of the bottom baffle on the mass transfer coefficient and the gas pressure drop in the WSA at different liquid-phase circulation rates was investigated. The results are shown in Figures 6 and 7. At higher liquid flow rates $\left(2.0 \mathrm{~m}^{3} / \mathrm{h}\right.$ and $\left.2.5 \mathrm{~m}^{3} / \mathrm{h}\right)$, the addition of baffles has no significant effect on volumetric mass transfer coefficient and gas pressure drop of the WSA. However, at the lower liquid flow rate $\left(1.5 \mathrm{~m}^{3} / \mathrm{h}\right)$, the bottom baffle has a significant effect on the volumetric mass transfer coefficient as the gas velocity increases. In the experimental system, the value increases of up to $13 \%$ comprised to the traditional structure (as shown in Figure 1a). Meanwhile, the pressure drop also has a certain increase in various gas inlet velocities, and its value increases by $6 \%$ to $10 \%$. This may be due to the addition of the baffle to enhance the gas-liquid interaction in the WSA.

The results of experimentally measured reflux ratio are shown in Figure 8. The bottom baffle has a smaller effect on the reflux ratio at higher liquid circulation rates. However, at low liquid-phase circulation flow rates, the addition of the baffle makes the reflux ratio increase at each gas velocity. That indicates that the addition of baffle changes the gas-liquid two-phase interaction state in the WSA and makes the axial velocity upward in the gas phase. Therefore, more liquid phase is involved under this flow state. At the lower liquid circulation flow rate, a large number of wall-attached atomization cause most of the liquid at the liquid seal to stick against the inner wall of the main cylinder, and the liquid-filled liquid surface is depressed downward at the axial center to form a gas phase. The addition of baffles restricts the tangential velocity at the liquid seal vortex and increases the axial velocity and radial velocity components, i.e., original rotational motion turns into a vertical tumbling motion. This not only accelerates the update of the gas-liquid two-phase contact area on the surface of the liquid seal, but also causes part of the gas in the center of the vortex to be entrained in the liquid phase, resulting in a large number of bubbles, which causes the gas-liquid two phases to remix and increase the mass transfer area, thereby improving the mass 
transfer performance. The violent tumbling movement of the liquid and the generation of air bubbles make the degree of instability of the surface of the liquid seal intensify, and the

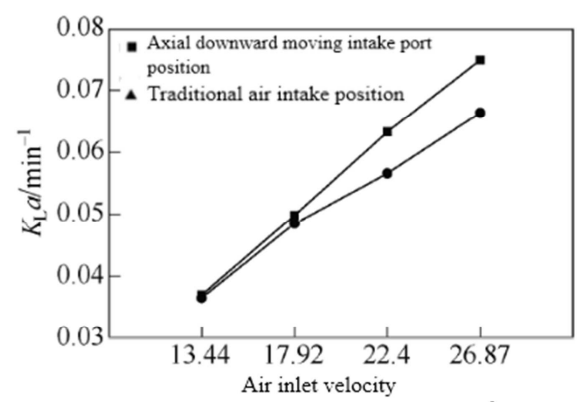

(a) Liquid circulation flow $1.5 \mathrm{~m}^{3} / \mathrm{h}$

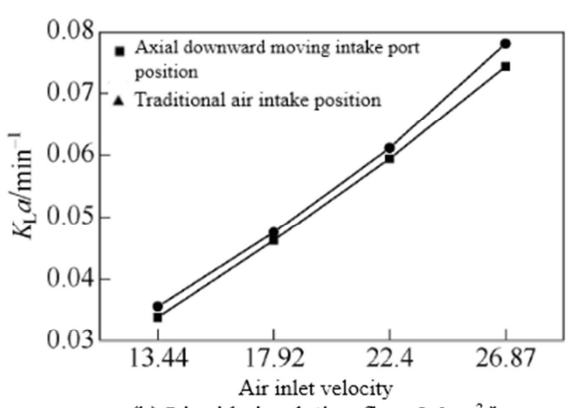

(b) Liquid circulation flow $2.0 \mathrm{~m}^{3} / \mathrm{h}$ liquid is more likely to be stripped and extracted by the rising swirling flow field, resulting in an increase in the liquid-phase reflux ratio.

Figures 6. Effect of bottom baffle on gas-liquid mass transfer coefficient in WSA under different liquid flow rates.

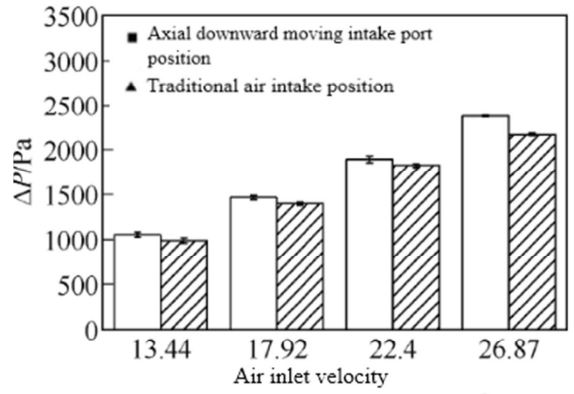

(a) Liquid circulation flow $1.5 \mathrm{~m}^{3} / \mathrm{h}$

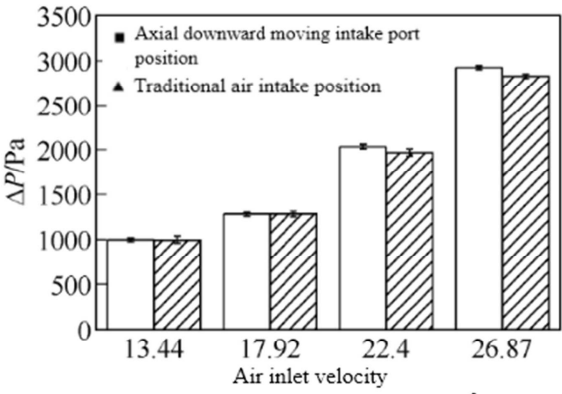

(b) Liquid circulation flow $2.0 \mathrm{~m}^{3} / \mathrm{h}$

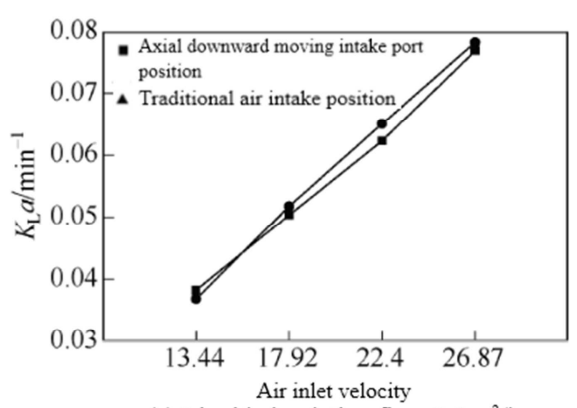

(c) Liquid circulation flow $2.5 \mathrm{~m}^{3} / \mathrm{h}$

Figures 7. Effect of bottom baffle on gas pressure drop in WSA under different liquid flow rates.

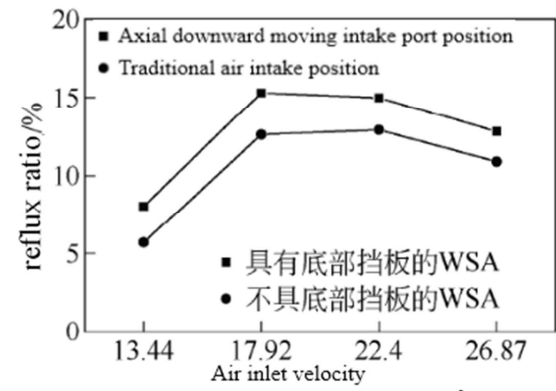

(a) Liquid circulation flow $1.5 \mathrm{~m}^{3} / \mathrm{h}$

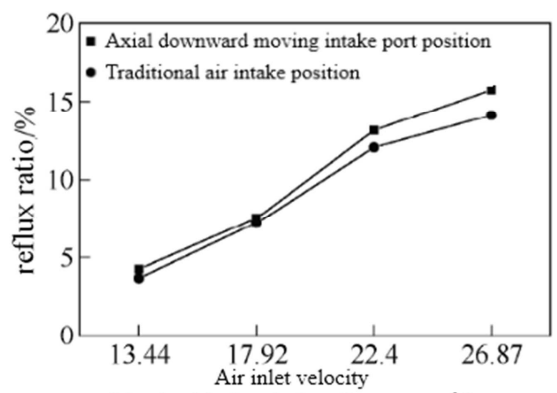

(b) Liquid circulation flow $2.0 \mathrm{~m}^{3} / \mathrm{h}$

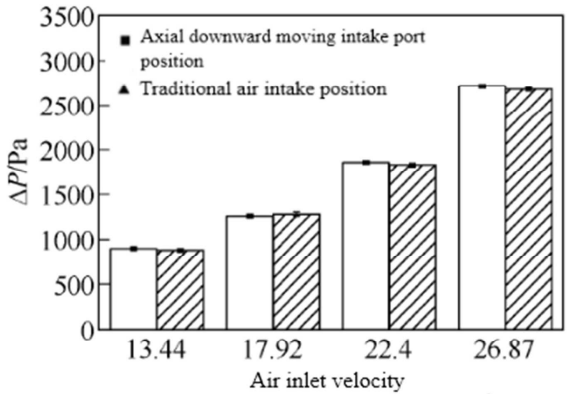

(c) Liquid circulation flow $2.5 \mathrm{~m}^{3} / \mathrm{h}$

Figures 8. Effect of bottom baffle on reflux ratio in WSA under different liquid flow rates.

\section{Conclusion}

In this present work, the effects of the inlet position and the bottom baffle on the mass transfer process of WSA were investigated by blowing off ammonia mass transfer experiments. The results show that the gas-phase inlet does not significantly increase the gas-liquid mass transfer performance in the WSA, but it can effectively reduce the internal gas-phase pressure drop, which is about $10 \%$. The baffle in the liquid seal area at the bottom of the WSA main cylinder can improve the gas-liquid mass transfer effect in the WSA under low liquid flow rate, and the effect is more significant with the increase of the inlet velocity. The mass transfer efficiency can be maximized in the experimental range. The increase is about $13 \%$.

\section{Acknowledgements}

The study was supported by the National Natural Science Foundation of China (21176273) and Chongqing Municipal Solid Waste Resource Utilization \& Treatment Collaborative Innovation Center Project (Shljzyh2017-003).

\section{References}

[1] Y. Lin, L. I. Lin, C. Zhang and X. U. Huasheng (2014) Progress on the mixing of liquid jet injected into a Crossflow. Acta Aeronautica Et Astronautica Sinica, 35, 46-57.

[2] X. Li, M. C. Soteriou (2018) Detailed numerical simulation of liquid jet atomization in crossflow of increasing density. International Journal of Multiphase Flow, 104, 214-232. 
[3] Y. Xia, L. Khezzar, M. Alshehhi, Y. Hardalupas (2018) Atomization of impinging opposed water jets interacting with an air jet. Experimental Thermal \& Fluid Science, 93, 11-22.

[4] Z. L. Cheng, X. J. Quan, L. Dai, Y. P. Yan and Q. H. Zhao (2013) Optimization of spray hole distribution in water-sparged aerocyclone. CIESC Journal, 64, 3182-3188.

[5] X. J. Quan, Q. H. Zhao, J. X. Xiang, Z. L. Cheng and F. P. Wang (2012) Mass transfer mechanism of a water-sparged aerocyclone reactor. Advanced Materials Research, 398-398, 279-283.

[6] X. J. Quan, Z. L. Cheng, F. Xu, F. C. Qiu, L. Dai and Y. P. Yan (2014) Structural optimization of the porous section in a water-sparged aerocyclone reactor to enhance the air-stripping efficiency of ammonia. Journal of Environmental Chemical Engineering, 2, 1199-1206.

[7] Q. H. Zhao, X. J. Quan, J. X. Xiang, F. P. Wang and Z. L. Cheng (2011) Gas phase pressure drop characteristics in a water-sparged aerocyclone. CIESC Journal, 62, 2507-2511.

[8] Q. H. Zhao, X. J. Quan, Z. L. Cheng and Y. P. Yan (2013) Wet desulfurization of flue gas and mass transfer mechanism in water-sparged aerocyclone. CIESC Journal, 64, 3993-4000.
[9] X. J. Quan, F. P. Wang, Q. H. Zhao, T. T. Zhao and J. X. Xiang (2009) Air stripping of ammonia in a water-sparged aerocyclone reactor. Journal of Hazardous Materials, 170, 983-988.

[10] Z. L. Cheng, X. J. Quan, Y. P. Yan, L. Dai (2014) Jet flow pattern and its effects on mass transfer area and gas phase pressure drop in a water-sparged aerocyclone. CIESC Journal, 65, 2914-2920.

[11] Z. L. Cheng, F. Xu, X. J. Quan, F. C. Qiu and M. X. Dai (2015) Effect of diameter and length of vortex finder in water-sparged aerocyclone reactor on its mass transfer performance in air stripping of ammonia. CIESC Journal, 66, 1642-1648.

[12] C. Matter-Müller, W. Gujer and W. Giger (1981) Transfer of volatile substances from water to the atmosphere. Water Research, 15, 1271-1279.

[13] Y. Su, T. Ng, Y. Zhang, J. H. Davidson (2018) A mesoscopic model for transient mass transfer of volatile organic compounds from porous walls of different structures. International Journal of Heat \& Mass Transfer, 117, 36-49. 